\title{
Erbin loss promotes cancer cell proliferation through feedback activation of Akt-Skp2-p27 signaling
}

Hao Huang ${ }^{\mathrm{a}, \mathrm{b}, 1}$, Yuhua Song ${ }^{\mathrm{c}, 1}$, Yan Wu ${ }^{\mathrm{a}, 1}$, Ning Guo ${ }^{\mathrm{a}}$, Yuanfang Ma ${ }^{\mathrm{b}, *}$, Lu Qian ${ }^{\mathrm{a},{ }^{*}}$

${ }^{a}$ Department of Pathophysiology, Institute of Basic Medical sciences, Beijing 100850,

P.R. China

${ }^{\mathrm{b}}$ Laboratory of Cellular and Molecular Immunology, Medical School of Henan

University, Kaifeng 475004, P.R. China.

${ }^{\mathrm{c}}$ The Affiliated Hospital of Medical College, Qingdao University, Qingdao, PR China.

Keywords: Erbin; Akt; Skp2; p27; cell proliferation; tumorigenesis

${ }^{1}$ These authors contributed equally to this work.

*Authors for correspondence (qianlubj@163.com ; mayf@henu.edu.cn) 


\section{Abstract}

Erbin localizes at the basolateral membrane to regulate cell junctions and polarity in epithelial cells. Dysregulation of Erbin has been implicated in tumorigenesis, and yet it is still unclear if and how disrupted Erbin regulates the biological behavior of cancer cells. We report here that depletion of Erbin leads to cancer cell excessive proliferation in vitro and in vivo. Erbin deficiency accelerates S-phase entry by down-regulating CDK inhibitors p21 and p27 via two independent mechanisms. Mechanistically, Erbin loss promotes p27 degradation by enhancing E3 ligase Skp2 activity though augmenting Akt signaling. Interestingly, we also show that Erbin is an unstable protein when the Akt-Skp2 signaling is aberrantly activated, which can be specifically destructed by SCF-Skp2 ligase. Erbin loss facilitates cell proliferation and migration in Skp2-dependent manner. Thus, our finding illustrates a novel negative feedback loop between Erbin and Akt-Skp2 signaling. It suggests disrupted Erbin links polarity loss, hyperproliferation and tumorigenesis. 


\section{Introduction}

Erbin is a novel LAP (for leucine-rich repeat and PDZ) family member that was originally characterized as an ErbB2-interacting protein [1]. LAP proteins are involved in the establishment of epithelial cell polarity, by localizing at the basolateral membrane of epithelia [2]. Recent studies suggest a role for LAP protein such as scribble in human cancers. For instance, scribble is frequently deregulated in human colorectal, breast, prostate and endometrial cancers $[3,4,5,6]$. Loss of scribble disrupts cell polarity, inhibits apoptosis, and induces cell hyperproliferation that correlates with tumor onset and progression [3,5,6]. Conditional knockout mice reveal that scribble deficiency promotes prostate neoplasia [3]. These studies establish scribble as a tumor suppressor and present polarity proteins as key regulators in tumorigenesis. Erbin, an epithelial-enriched protein, is also localized basolaterally and proposed as a mediator of basolateral trafficking, over the past decades, the polarity role(s) assigned to Erbin has been increasingly suggested. Erbin has been shown to directly bind to p120 catenin family members (p00071 and $\delta$-catenin) and hemidesomosomal protein bullous pemphigoid antigen 1 (BPAG1), integrin $\beta 4$ subunit as well as adhesion molecules E-cadherin $[1,7,8]$. These interaction data strongly suggest that Erbin is linked to cytosketon-associated protein complex and involved in the establishment and the maintenance of cell-cell and cell-basement membrane adhesion.

Erbin has also been reported as negative regulators involved in diverse signaling pathways [1]. Erbin inhibits Ras-mediated activation of MAPK signaling by binding Sur-8 and disrupting the Sur-8-Ras-Raf complex [9]. It interacts with Nod2 and 
suppresses the Nod2-dependent NF- $\kappa \mathrm{B}$ activation [10]. Erbin has also been shown to block oligomerization of Smads and affect their transcriptional output [11]. Accordingly, Erbin is clearly implicated in regulating many aspects of biological behaviors of cancer cells such as cellular proliferation, apoptosis and inflammatory response. A recent study form our group demonstrated that Erbin is significantly decreased or lost in cervical cancer tissues and loss of Erbin endows cervical cancer with resistance to anoikis by inhibiting STAT3 signaling [12]. Of note is that other group recently found that Erbin plays a positive role in ErbB2-dependent breast tumor growth [13]. In this regard, the role of Erbin in human cancer is conflicting. However, neither the potential function of Erbin plays during tumorigenesis nor the mechanism by which Erbin is deregulated is well understood.

We here demonstrate that depletion of Erbin markedly promotes cervical cell proliferation and tumor outgrowth by accelerating cell cycle progression from G1 to S phase though down-regulating the CDK inhibitors p27 and p21 expression. Furthermore, we reveal that Erbin loss induces aberrant activation of Akt-Skp2 axis, promoting p27 destruction. In turn, Erbin protein is specifically recognized and degraded by SCF Skp2 ubiquitin ligase, indicating a feedback loop between Akt-Skp2 signaling and Erbin during cancer. These observations not only first reveal a novel regulatory mechanism for the dysregulation of Erbin expression but also underscore its tumor-suppressor function. 


\section{Materials and Methods}

\section{Cell culture, treatment and reagents}

HeLa cells were incubated in DMEM containing 10\% fetal bovine serum (FBS) (Life Technologies). For half-life studies, cycloheximide $(20 \mu \mathrm{g} / \mathrm{ml}, \mathrm{CHX}$, Sigma) was added to medium $40 \mathrm{~h}$ after transfection for indicated time points. For nocodazole release experiments, cells were blocked with $330 \mathrm{mM}$ nocodazole (Sigma) for $18 \mathrm{~h}$ to trap the cells at prometaphase. Mitotic cells were collected by mechanical shake-off, washed and released into medium. For detection of Skp2 acetylation, cells were pretreated with $1 \mathrm{mM}$ trichostatin A (TSA) and $5 \mathrm{mM}$ nicotinamide (NAM) for $2 \mathrm{~h}$ to block the histone deacetylases (HDAC) activity.

\section{Plasmids, siRNA, transfection and viral infection}

The plasmid Myc-Erbin was kindly provided by Dr. Lin Mei (Institute of Molecular Medicine and Genetics, Medical College of Georgia). To construct Flag-27, Flag-p21 and Flag-Skp2, the respective ORFs were amplified by PCR and subcloned in frame into pCMV-flag. The siRNA duplexes targeting Erbin, Skp2 and PTEN were designed and synthesized by Ribio Biotech (Guangzhou, China) (for siRNA sequences see Supplementary Materials).

Plasmid transfection was carried out using Fugene HP (Roche). siRNA transfection was carried out using Lipofectamine ${ }^{\mathrm{TM}}$ RNAiMAX (Invitrogen). Erbin-shRNA was constructed and packaged by the GeneChem Company (Shanghai, China), and then transduced into the HeLa cells by lentiviral infection.

\section{Immunoblotting and immunoprecipitation}

Cells were lysed with RIPA buffer and then subjected for immunoblotting according 
to the standard protocols. For immunoprecipitation, cell pellets were suspended in IP buffer $(50 \square \mathrm{mM}$ Tris $\mathrm{HCl}$ at $\mathrm{pH} 7.5,150 \square \mathrm{mM} \mathrm{NaCl}, 1 \square \mathrm{mM}$ EDTA, $1 \square \mathrm{mM} \mathrm{MgGl}$ 0.5\% Triton $\mathrm{X}-100$ ) and incubated with the appropriate antibody for $4 \mathrm{~h}$ at $4^{\circ} \mathrm{C}$ followed by incubation for $2 \mathrm{~h}$ with protein $\mathrm{A} / \mathrm{G}$ beads (Santa cruz). IP complexes were washed six times with IP buffer before being resolved by SDS-PAGE and immunoblotted with the indicated antibodies. Details of antibodies used are given in Supplementary Materials.

\section{Real-Time PCR}

RNA was extracted with the Tripure kit, and the reverse transcription reaction was performed with RevertAidTM First Strand cDNA Synthesis Kit. Real-time PCR was performed using SYBR green PCR core reagent (Applied Biosystems) on Real-Time PCR Detection System (Stratagene $\mathrm{M} \times 300 \mathrm{P})$ in triplicate.

\section{Cell proliferation and cell clonogenic assay}

Cells were seeded into 24 -well plates with 5000 cells per well in triplicate. The ATPlite (Vigras, China) cell proliferation assay and Cell-Light EdU DNA Cell Proliferation Kit (Ribobio, China) were performed according to the manufacturer's specifications. For clonogenic assay, cells were seeded into 6-well plates (150 cells per well) in triplicate and cultured for 10 days. The colonies on the plates were fixed with $4 \%$ paraformaldehyde and stained with crystal violet. Colonies with more than 50 cells were counted. The representative results of three independent experiments with similar trend were presented.

\section{Wound healing assay}


Confluent HeLa cell monolayers were wounded by scraping once horizontally and vertically with a $100 \mu$ pipet tip and further incubated in DMEM with $1 \%$ FBS. The relative area of the tumor cell covered was calculated at various time points. The experiment was performed by duplicate.

\section{Immunohistochemistry}

Thirty invasive ductal carcinoma tissues were obtained from 307 Hospital, Beijing, China, with the informed consent of patients and approval for the experiments from 307 Hospital. Immunohistochemistry (IHC) staining was performed as previously described [12].

\section{In vivo assays for tumor growth}

Six-week old female Balb/C athymic nude mice were purchased from Beijing Vital River Laboratory Animal Technology Co. Ltd. 1 X10 ${ }^{6} \mathrm{HeLa} / \mathrm{Ctrl}$ shRNA or HeLa/Erbin-sh RNA cells were subcutaneously injected into the right flanks of each mouse. Each group contains six mice. The mice were killed after 30 days and the tumors were dissected and weighted.

\section{Statistical analysis}

Data were presented as the mean \pm SE. The statistical analysis was performed with Student's $t$-test. $p<0.05$ was considered as statistically significant.

\section{Results}

\section{Erbin knockdown promotes cervical cancer cell proliferation through down-regulating p21 and p27.}


As our previous study shows that Erbin is significantly downregulated in cervical cancer tissues [12], we sought to determine whether Erbin, like another LAP family member scribble, is involved in signal transduction to negatively regulate cell proliferation. We first used two independent siRNAs to silence the expression of Erbin in human cervical carcinoma cell line HeLa and then examined the effect of Erbin deficiency on cell proliferation by monitoring ATP levels using the ATPLite assay. Both two independent siRNAs efficiently silenced the expression of Erbin in HeLa cells, resulting in significantly increased cell proliferation (Fig. 1A). To confirm the pro-proliferative role of Erbin deficiency, we established the stable Erbin-knockdown HeLa cells using lentiviral Erbin shRNA vector (Fig. 1B) and assessed the effects of Ebin knockdown on colony formation and tumor growth potentials. Consistently, the colony formation ability was remarkably increased after Erbin stably knockdown (Fig. 1C and 1D). We then inoculated cells with control and Erbin knockdown subcutaneously into the flanks of nude mice, and monitored the tumor burden of xenografts after 30 days. Expectedly, there was a more than 2-fold increase in tumor size and weight upon Erbin deficiency (Fig. 1E and 1F), indicating Erbin loss facilitates the growth of human cervical cancer of xenografts.

We next examined the cell cycle distribution of Erbin-depleted HeLa cells and observed the expression of key regulators for cell cycle progression. As shown in Fig. $1 \mathrm{G}$ and Fig. S1A, at $48 \mathrm{~h}, 72 \mathrm{~h}$ and $96 \mathrm{~h}$ post-transfection, a significant increase in the S-phase population was observed in Erbin-depleted cells. Correspondingly, Erbin knockdown prominently resulted in the down-regulation of cyclin-dependent kinase 
inhibitors, p21 (Cip1) and p27 (Kip1), although the level of cyclin D was slightly increased. Both p21 and p27 play an essential role in the negative regulation of progression through $\mathrm{G} 1$ to $\mathrm{S}$ phase in mammalian cells [14]. When p21 or p27 expression was restored to its baseline level in Erbin-depleted cells though transient transfection of moderate Flag-tagged p21 or p27 (Fig. S1C), we found that the increase in Edu-labelled cells by Erbin depletion was almost completely blocked by exogenous p21 or p27 (Fig. 1H and 1I), indicating that Erbin loss promotes S-phase progression though modulating p21 and p27 expression. We also detected the p21 and p27 mRNA levels upon Erbin knockdown and found that p21 mRNA levels were significantly reduced after Erbin knockdown, while there was no change for p27 mRNA levels (Fig. 1J), suggesting the regulations are via two separate mechanisms.

\section{Erbin knockdown enhances p27 degradation though Akt-dependent Skp2}

\section{activation.}

The abundance of p27 is thought to be controlled mainly at the level of its post-transcriptional degradation via Skp2 [15]. Skp2 forms a Skp2-SCF complex with Skp1, Cullin-1 (Cul-1), and RBx1 to constitute an E3 ligase activity that triggers p27 ubiquitination and degradation [16]. To address the possibility that Skp2 functions in p27 down-regulation by Erbin knockdown, we first accessed the half-life of endogenous Skp2 after Erbin knockdown using the cycloheximide (CHX) chase assay. It was shown that Erbin depletion clearly prolonged the half-life of endogenous Skp2 and concomitantly accelerated degradation of its downstream target p27, whereas 
didn't affect the APC/C target Cdc20 (Fig. 2A and 2B). Erbin depletion also markedly enhanced the half-life of exogenously expressed Flag-Skp2 in HeLa cells (Fig. 2C), indicating that Erbin specifically regulates Skp2 stability.

To confirm whether Skp2 indeed mediates the role of Erbin in p27 turnover, we first chose three single Skp2 siRNAs to validate their knockdown efficiencies and found that and siRNA ${ }^{\# 2}$ and siRNA \#3 could efficiently silence Skp2 expression and result an increase in p27 abundance (Fig. 2D). We knocked down Erbin, Skp2 or both in G2/M synchronized cells with nocodazole and monitored the expression of Skp2-p27 during G1-S progression (release after nocodazole block). In cells transfected with control siRNA, Skp2 protein levels fell and p27 levels rose after release. Erbin knockdown resulted in a more rapid p27 turnover, whereas Skp2 knockdown abrogated the effects of Erbin knockdown on p27 destruction (Fig. 2E), indicating a link between the Skp2-p27 axis and Erbin during cell cycle.

As Akt-mediated Skp2 phosphorylation and acetylation stabilize Skp2 expression and enhances its E3 ligase activity [17,18], we explored the regulatory role of Erbin in Akt signaling. We found that knockdown of Erbin markedly augmented EGF-induced phosphorylation of Akt at Ser473 and Ser308, whereas did not influence ERK activation (Fig. 2F). We also noticed that Erbin knockdown increased Skp2 protein levels and remarkably suppressed mitogen-induced p27 expression. Accordingly, Erbin regulates Skp2-p27 signaling probably through an Akt-dependent pathway independently of ERK activation. Using anti-phospho-Akt substrate antibody following Skp2 immunoprecipitation, we confirmed that Skp2 is a specific 
phospho-substrate for Akt, as shown in Fig.2 G, endogenous Skp2 phosphorylation in HeLa cells was drastically repressed by the PI3K/Akt inhibitor, LY294002, but not the ERK inhibitor, U0126. Furthermore, we found that Erbin knockdown enhanced EGF-stimulated Skp2 phosphorylation and the association of Skp2 and Skp1 (Fig. 2H). Intriguingly, using anti-pan-acetylated Lysine antibody (anti-Ac-K), we also found that Erbin knockdown promoted the acetylation of endogenous Skp2 and formation of Skp2-Skp1 complex (Fig. 2I). These results strongly imply that Erbin play a negative role in Akt-dependent phosphorylation and acetylation regulation of the SCF-Skp2 function. Although it has been reported that Akt1 activation tigers Skp2 cytoplasmic retention [18,19], Erbin depletion-induced enhancement of Akt activation failed to do so. Immunofluorescence revealed that Skp2 was exclusively in the nuclear compartment and depletion of Erbin had no significant impact on its subcellular distribution (Fig. S2), suggesting that Skp2 is not robustly regulated by Akt1 for its cytoplasmic localization in HeLa cells.

\section{Erbin expression is negatively regulated by a feedback Akt-Skp2 signaling}

Furthermore, we explored the underlying mechanisms that may contribute to dysregulation of Erbin. It was noticed that Erbin expression was rapidly decreased following the addition of CHX (Fig. 2A), suggesting that Erbin may be regulated in a proteasomal degradation manner. Surprisingly, knockdown of Skp2 resulted in a marked increase of Erbin expression and accumulation of p27 abundance (Fig. 3A), raising the possibility that Skp2 modulates Erbin protein stability though direct 
SCF-Skp2 ubiquitin ligase. In support of this, depletion of Skp2 clearly suppressed the endogenous and exogenous Erbin degradation in CHX chase assay (Fig. 3B, C); moreover, Skp1 or Cul1 knockdown profoundly increased Erbin abundance and stability (Fig. S3). We further found that when HA-Erbin and Flag-Skp2 co-expressed in 293 T cells, HA-Erbin strongly interacted with Flag- Skp2 (Fig. 3D), although endogenous association of Erbin and Skp2 was not detected. Then in vivo ubiquitination assay, the ubiquitination of Erbin was moderately enhanced by Skp2 overexpression (Fig. 3E). These data support the regulatory role of Skp2 in Erbin dysregulation and proposed an important feedback loop composed of Erbin and Skp2. Akt1 kinase modulates Skp2 E3 ligase activity. We further determined a presumed role of Akt signaling in Erbin expression. We monitored the effect of PI3K/Akt inhibition by LY294002 on Erbin expression and found that a significant increase of levels of Erbin and p27 upon Akt inactivation (Fig. 3F). In contrast, PTEN siRNA-mediated Akt activation in DU145 cells led to a rapid degradation of Erbin (Fig. 3G). These results suggested that PI3K/Akt pathway involved in Skp2-mediated Erbin expression.

\section{Loss of Erbin cooperates with Skp2 to promote cell migration}

Skp2 is overexpressed in numerous human cancers[16]. We next investigate whether Skp2 overexpression correlates with Erbin loss in clinical cancer samples. Initially, we examined the expression of Skp2 and Erbin in 30 invasive ductal carcinoma samples by immunohistochemistry. Sequential sections showed low or absent Erbin 
staining appeared in Skp2-high tumors and vise visa (Fig. 4A). Considering both staining intensity and area, Erbin and Skp2 staining were categorized to three levels (low, medium and high). Of 13 Skp2-low samples, 11 sections showed medium and high levels of Erbin, whereas of Skp2-high samples, 50\% showed low or absent Erbin (Fig. 4B). Erbin expression negatively correlated with Skp2 levels in limited breast tumor samples. This suggests that loss of Erbin may potentiate pro-oncogenic function in coordination with Skp2 overexpression. Given that Erbin has well been implicated in cell polarity and tissue architecture and Skp2 have a function in tumor invasion and metastasis $[1,16]$, we examined whether Erbin may have a role in cell migration, which underlies these steps in tumor progression. In wound scratch assay as shown in Fig. 4C, Erbin knockdown significantly accelerated wound closure, whereas Skp2 knockdown remarkably delayed wound; the effect of Erbin knockdown on cell migration was completely reversed by Skp2 knockdown. Our results underlie that loss of Ebin may contribute to Skp2 function, thereby promoting caner progression by regulation of cell proliferation, cell survival as well as cell migration.

\section{Discussion}

In recent years, a large body of evidences supports the contention that over-activation of Skp2-p27 axis positively correlates with tumor malignancy and poor diagnosis[16]. Erbin dysregulation has previously been reported in cervical tumors, colon carcinomas and breast carcinomas $[12,13,20]$. Here, we provide evidence to support the mechanistic connection between Erbin loss and aberrant Skp2-p27 axis, which 
coordinately drives cancer cells to uncontrolledly proliferate through cell cycle regulation. Recent findings show that Akt, a major kinase that coordinates diverse signaling pathway, directly phosphorylates or/and indirectly acetylates Skp2, thus activating SCF-Skp2 $[17,18,19]$. Our previous data shows that downregulation of Erbin in Her2-overexpressiong breast cancer cells induces trastuzumab resistance through enhancement of Akt activation [20]. Consistently, our present data reveals that Erbin loss augments mitogen-induced Akt activation, which appears to be responsible for the enhanced Skp2 phosphorylation, acetylation and SCF-Skp2 activity, as well as p27 destruction. We for the first time demonstrate Erbin acts as a negative modulator to regulate the Akt1-Skp2-p27 signaling pathway (Fig.7 E). More interestingly, we reveal that Erbin itself is unstable protein and degraded by SCF-Skp2 complex, while Aberrant Akt-Skp2 signaling in turn promotes Erbin destruction. Our preliminary data shows that the high Skp2 correlates with low Ebin levels in invasive ductal breast cancer samples and loss of Erbin leads to a rapid wound healing in a Skp2-dependent manner. Together, these data suggest that Erbin loss and Skp2 overexpression may be mutually and causally connected in human cancers. It is also possible that disrupted Erbin expression may facilitate Skp2 oncogenic function. Our findings provide novel understanding how LAP protein Erbin disruption have an impact on cell proliferation and oncogenic signal transduction pathway. However, further studies are warranted to better understand the biological significance and mechanisms of this complicated negative feedback loop between Erbin and Skp2. 


\section{Acknowledgements}

This work was supported by National Basic Research Program of China (973 Program, No. 2010CB911904) and National Science Foundation of China (No. 31271440).

\section{References}

[1] L. Dan, M. Shi, H. Duan, C. Han, N. Guo, Erbin, a negative regulator in diverse signal pathways, Curr Protein Pept Sci 11 (2010) 759-764.

[2] P.J. Bryant, A. Huwe, LAP proteins: what's up with epithelia?, Nat Cell Biol 2 (2000) E141-143.

[3] H.B. Pearson, P.A. Perez-Mancera, L.E. Dow, A. Ryan, P. Tennstedt, D. Bogani, I. Elsum, A. Greenfield, D.A. Tuveson, R. Simon, P.O. Humbert, SCRIB expression is deregulated in human prostate cancer, and its deficiency in mice promotes prostate neoplasia, J Clin Invest 121 (2011) 4257-4267.

[4] Z. Ouyang, W. Zhan, L. Dan, hScrib, a human homolog of Drosophila neoplastic tumor suppressor, is involved in the progress of endometrial cancer, Oncol Res 18 (2010) 593-599.

[5] M.E. Feigin, S.D. Akshinthala, K. Araki, A.Z. Rosenberg, L.B. Muthuswamy, B. Martin, B.D. Lehmann, H.K. Berman, J.A. Pietenpol, R.D. Cardiff, S.K. Muthuswamy, Mislocalization of the cell polarity protein scribble promotes mammary tumorigenesis and is associated with basal breast cancer, Cancer Res 74 (2014) 3180-3194. 
[6] Y. Kamei, K. Kito, T. Takeuchi, Y. Imai, R. Murase, N. Ueda, N. Kobayashi, Y. Abe, Human scribble accumulates in colorectal neoplasia in association with an altered distribution of beta-catenin, Hum Pathol 38 (2007) 1273-1281.

[7] F. Jaulin-Bastard, J.P. Arsanto, A. Le Bivic, C. Navarro, F. Vely, H. Saito, S. Marchetto, M. Hatzfeld, M.J. Santoni, D. Birnbaum, J.P. Borg, Interaction between Erbin and a Catenin-related protein in epithelial cells, J Biol Chem 277 (2002) 2869-2875.

[8] I. Izawa, M. Nishizawa, Y. Tomono, K. Ohtakara, T. Takahashi, M. Inagaki, ERBIN associates with p0071, an armadillo protein, at cell-cell junctions of epithelial cells, Genes Cells 7 (2002) 475-485.

[9] Y.Z. Huang, M. Zang, W.C. Xiong, Z. Luo, L. Mei, Erbin suppresses the MAP kinase pathway, J Biol Chem 278 (2003) 1108-1114.

[10] C. McDonald, F.F. Chen, V. Ollendorff, Y. Ogura, S. Marchetto, P. Lecine, J.P. Borg, G. Nunez, A role for Erbin in the regulation of Nod2-dependent NF-kappaB signaling, J Biol Chem 280 (2005) 40301-40309.

[11] F. Dai, C. Chang, X. Lin, P. Dai, L. Mei, X.H. Feng, Erbin inhibits transforming growth factor beta signaling through a novel Smad-interacting domain, Mol Cell Biol 27 (2007) 6183-6194.

[12] Y. Hu, H. Chen, C. Duan, D. Liu, L. Qian, Z. Yang, L. Guo, L. Song, M. Yu, M. $\mathrm{Hu}$, M. Shi, N. Guo, Deficiency of Erbin induces resistance of cervical cancer cells to anoikis in a STAT3-dependent manner, Oncogenesis 2 (2013) e52.

[13] Y. Tao, C. Shen, S. Luo, W. Traore, S. Marchetto, M.J. Santoni, L. Xu, B. Wu, C. 
Shi, J. Mei, R. Bates, X. Liu, K. Zhao, W.C. Xiong, J.P. Borg, L. Mei, Role of Erbin in ErbB2-dependent breast tumor growth, Proc Natl Acad Sci U S A 111 (2014) E4429-4438.

[14] A.M. Abukhdeir, B.H. Park, P21 and p27: roles in carcinogenesis and drug resistance, Expert Rev Mol Med 10 (2008) e19.

[15] M. Pagano, S.W. Tam, A.M. Theodoras, P. Beer-Romero, G. Del Sal, V. Chau, P.R. Yew, G.F. Draetta, M. Rolfe, Role of the ubiquitin-proteasome pathway in regulating abundance of the cyclin-dependent kinase inhibitor p27, Science 269 (1995) $682-685$.

[16] G. Wang, C.H. Chan, Y. Gao, H.K. Lin, Novel roles of Skp2 E3 ligase in cellular senescence, cancer progression, and metastasis, Chin J Cancer 31 (2012) 169-177.

[17] Z. Wang, H. Inuzuka, J. Zhong, P. Liu, F.H. Sarkar, Y. Sun, W. Wei, Identification of acetylation-dependent regulatory mechanisms that govern the oncogenic functions of Skp2, Oncotarget 3 (2012) 1294-1300.

[18] T. Bashir, J.K. Pagan, L. Busino, M. Pagano, Phosphorylation of Ser72 is dispensable for Skp2 assembly into an active SCF ubiquitin ligase and its subcellular localization, Cell Cycle 9 (2010) 971-974.

[19] D. Gao, H. Inuzuka, A. Tseng, R.Y. Chin, A. Toker, W. Wei, Phosphorylation by Akt1 promotes cytoplasmic localization of Skp2 and impairs APCCdh1-mediated Skp2 destruction, Nat Cell Biol 11 (2009) 397-408.

[20] D. Liu, M. Shi, C. Duan, H. Chen, Y. Hu, Z. Yang, H. Duan, N. Guo, Downregulation of Erbin in Her2-overexpressing breast cancer cells promotes cell 
migration and induces trastuzumab resistance, Mol Immunol 56 (2013) 104-112.

\section{Figure legends}

Fig.1. Loss of Erbin promotes cervical cancer cell proliferation and down-regulates p21 and p27 expression. (A) HeLa cells were transfected with control or two independent Erbin siRNAs, harvested for immunoblotting (IB) analysis. Cell proliferation activities were assessed by the ATPlite assays at 24, 48, 72 and $96 \mathrm{~h}$ after siRNA transfection. Experiments were performed in triplicate and results are expressed as relative ATP levels (mean \pm SE of 3 determinations). (B) The establishment of stable Erbin-knockdown HeLa cells. (C, D) The colony formation ability was assessed. $* * P<0.01$. (E, F) In vivo xenograft assays for tumor growth. Six mice were studied per group. ${ }^{*} P<0.05$. (G) HeLa cells were transfected Erbin siRNA (\#2) or control siRNA for indicated time, at each time point, half of the cells was lysed and subjected to IB analysis, whereas the second half was harvested for cell cycle analysis by flow cytometry. Quantification of cell cycle phases is shown (bottom). (H, I) EdU incorporation assay in HeLa cells transfected with siRNA as indicated, along with Flag-p27 or Flag-p21 vectors. A typical experiment performed in triplicate was shown $($ Bar $=200 \mu \mathrm{M})$ and the percentage of EdU-positive cells was measured. (J) Real-time PCR analysis of p21 and p27 mRNA level after Erbin knockdown.

Fig.2. Erbin knockdown enhances Skp2-mediated p27 degradation though 
augmenting Akt signaling. (A and B) The half-life of endogenous Skp2 in HeLa cells with control and Erbin-knockdown. At $40 \mathrm{~h}$ after transfection, cells were treated with $\mathrm{CHX}$ at various time points, and harvested for IB analysis. Relative Skp2 expression was quantified. Skp2 band intensity was normalized to tubulin, then normalized to the $\mathrm{t}=0$ control siRNA group. (C) The exogenous Skp2 stability after Erbin knockdown. HeLa cells stably expressing Flag-tagged Skp2 were established and transfected with control or Erbin siRNA. The CHX chase experiment was performed as described above. (D) The efficacy of three independent Skp2 siRNAs was determined by immunoblotting. (E) The turnovers of Skp2 and p27 in synchronized HeLa cells. Cells were transfected with the indicate siRNA, synchronized by growth in nocodazole, and released into G1-S progression. The cells were harvested for IB analysis. (G) HeLa cells treated for 6 or $12 \mathrm{~h}$ with $20 \mu \mathrm{M}$ LY294002 or $20 \mu \mathrm{M}$ U1026 were harvested for immunoprecipitation (IP) with anti-Skp2 antibody, followed by immunoblotting with anti-Akt substrate phosho-antibody. (D) Skp2 phosphorylation in HeLa cells with control and Erbin-knockdown. Cells were serum-starved, treated with EGF at indicated time points, and harvested for IP assay followed by IB analysis. The association of Skp2 and Skp1 was also analyzed. (E) Skp2 acetylation in HeLa cells that were treated with EGF stimulation following indicate siRNA transfection. Where indicated, the cells were pretreated with TSA $(2 \mathrm{mM})$ and NAM $(10 \mathrm{mM})$ for $1 \mathrm{~h}$ before EGF treatment.

Fig.3. Erbin expression is specifically regulated by Skp2 SCF complex. (A) Skp2 
knockdown increased Erbin expression upon EGF stimulation. HeLa cells were transfected with control or Skp2 siRNA and then treated with EGF for indicated time points. The expression of indicated proteins was detected by IB analysis. (B) IB analysis of Erbin stability in HeLa cells with control and Skp2 knockdown. (C) IB analysis of exogenous HA-tagged Erbin protein stability after Skp2 knockdown. pEGFP-N1 was cotransfected to monitor transfection efficiency. (D) 293T cells were co-transfected with Flag-Skp2 and HA-Erbin, and harvested for co-IP assay followed by IB analysisn. (E) In vivo ubiquitination assay in $293 \mathrm{~T}$ cells transfected with HA-Erbin, Myc-Ub, along with or without Flag-Skp2. Cells were treated with $20 \mu \mathrm{m}$ MG132 or DMSO for $6 \mathrm{~h}$, and harvested for IP assay followed by IB analysis. Cell lysates were immunoprecipitated with anti-HA antibody and detected with the anti-Myc antibody. Whole cell lysates (Input) were subjected to immunoblotting to detect the expression of HA-Erbin and Flag-Skp2. (F) Inactivation of PI3K/Akt signaling enhanced Erbin expression. IB analysis of cell lysates from HeLa cells treated with the PI3K inhibitor LY294002 for the indicated time points. (G) PTEN siRNA-mediated Akt activation shortened the half-life of Erbin. DU145 cells were transfected with indicated siRNAs and treated with $\mathrm{CHX}$ for indicated times. Cell lysates were prepared and examined by immunoblotting with indicated antibodies.

Fig.4. Loss of Erbin potentiates pro-oncogenic function of Skp2. (A and B) Erbin expression is negatively correlated with Skp2 in human breast cancer. Representative breast cancer tissues stained for Erbin and Skp2 as examined by 
immunohistochemistry $\underline{(\text { Bar }=50 \mu \mathrm{M})}$. Both Erbin and Skp2 levels were classified as low, medium, or high based on the intensities of the IHC staining, and cases of patients classified in each category are depicted in the histogram. (C, D) Erbin knockdown enhances cell migration in Skp2 dependent manner. Confluent HeLa cells transfected with indicated siRNAs were re-plated for in vitro wound healing. The images of the wounds at different time points were captured $(\mathrm{Bar}=5 \mu \mathrm{M})$. Results are presented as mean \pm SE from two independent experiments. (E) A schematic representation of a negative feedback loop between Akt-Skp2 signaling and Erbin in tumor progression. 
Fig. 1

A
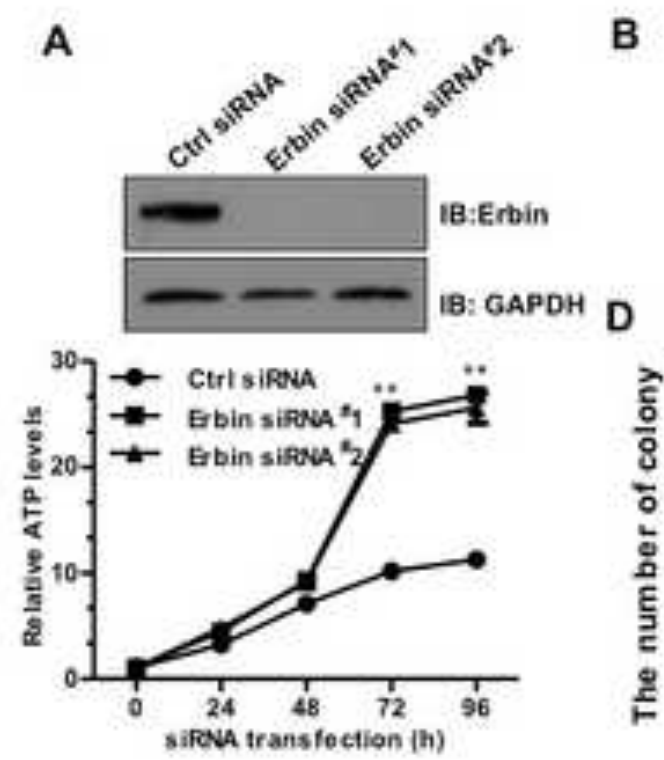

G

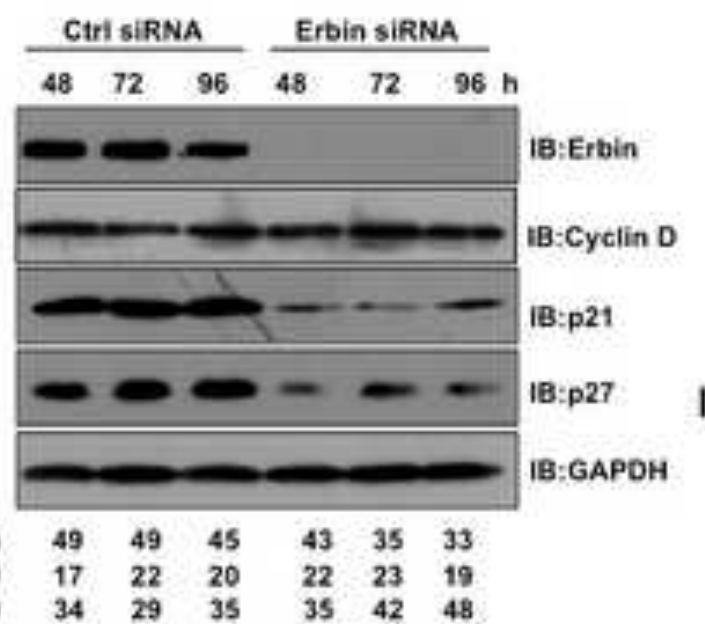

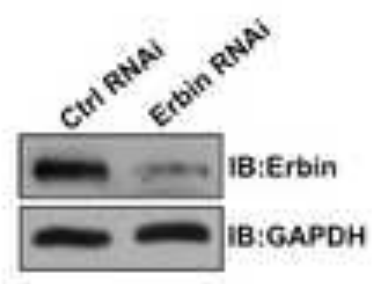

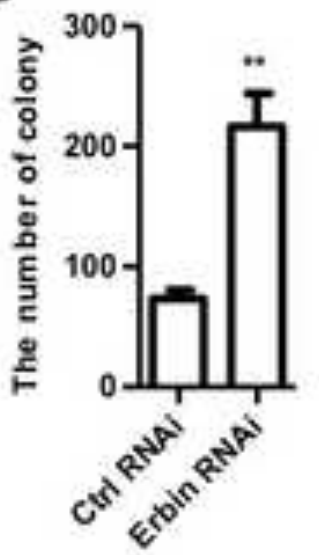

H
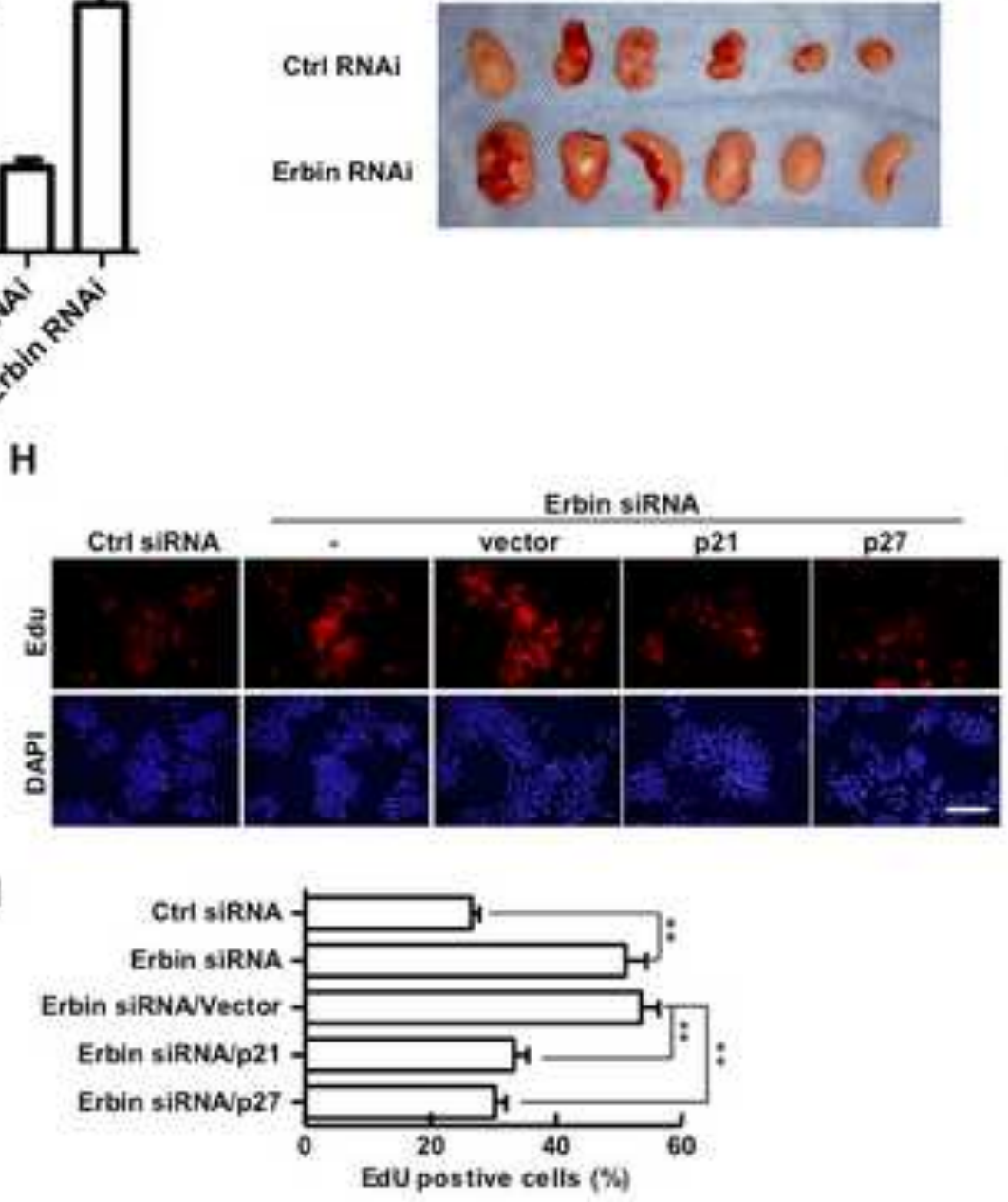

C

F

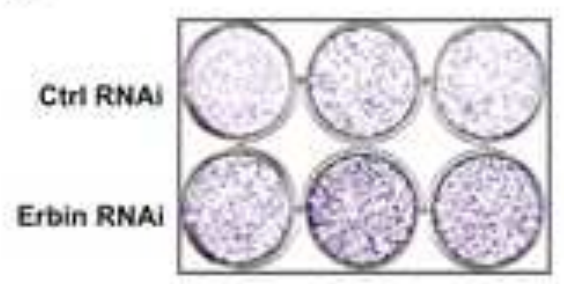

E

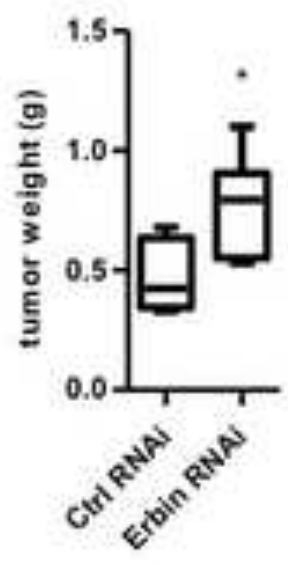

J

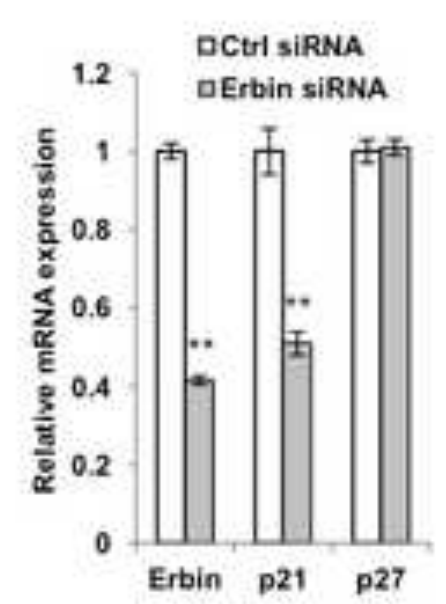


Fig. 2

A

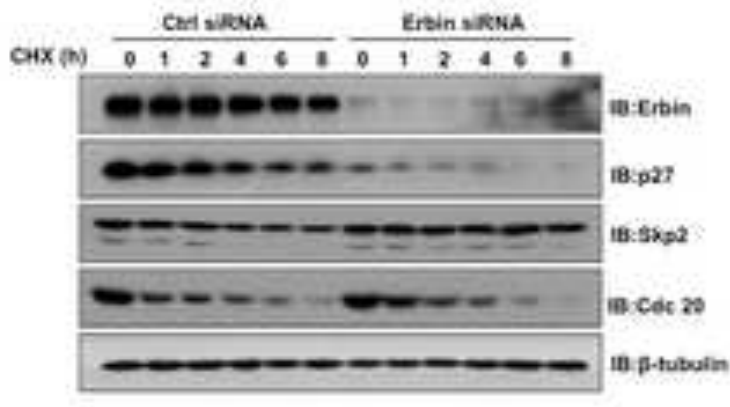

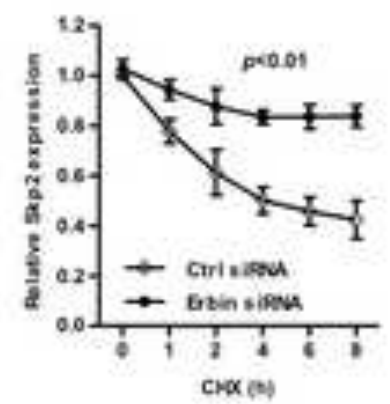

C

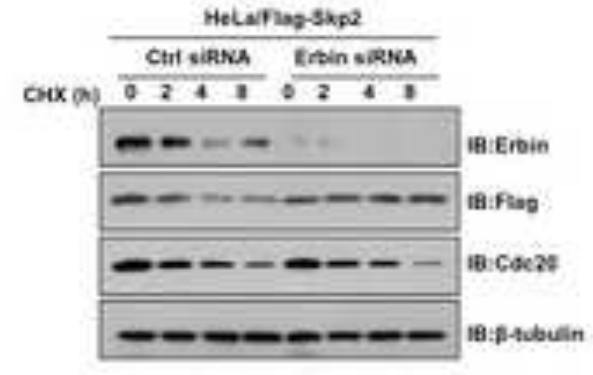

D

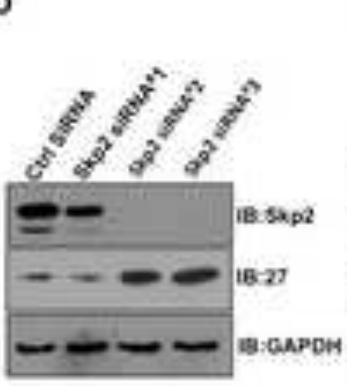

E

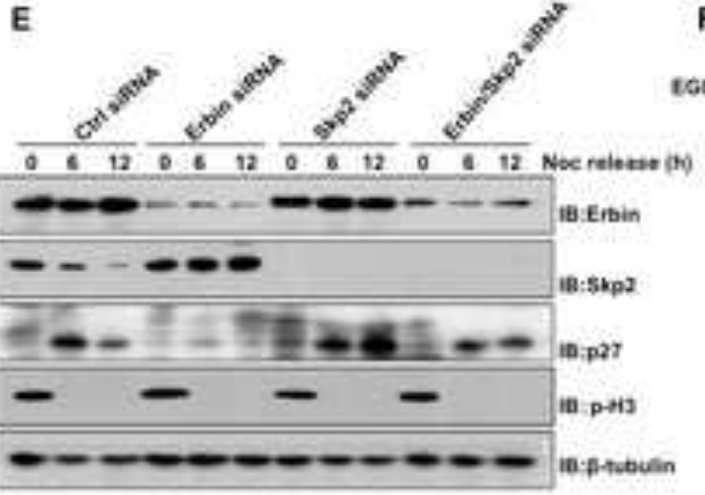

F

\begin{tabular}{|c|c|c|c|}
\hline \multicolumn{2}{|r|}{ COA SIBNA } & Ertin sRke & \multirow[b]{3}{*}{ mertin } \\
\hline 1 & 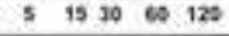 & $0 \quad \$ \quad 15300120$ & \\
\hline \multicolumn{3}{|c|}{ 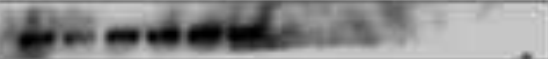 } & \\
\hline & -- & $m-\infty$ & It:p-Aks S173 \\
\hline & $-2-1$ & ----1 & in:p-ake T3on \\
\hline & 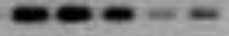 & 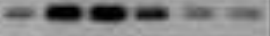 & 11:-p-tax \\
\hline & $=-2=-$ & $=2=2=0$ & IB: Sikge \\
\hline & $----\infty$ & $-\cdots-\cdots$ & 18:p2r \\
\hline & $-0--0-1$ & $-m-m-m$ & 18.ptutuine \\
\hline
\end{tabular}

G

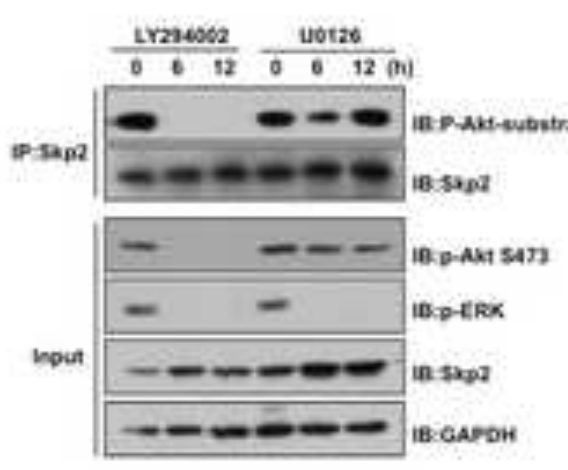

\section{H}
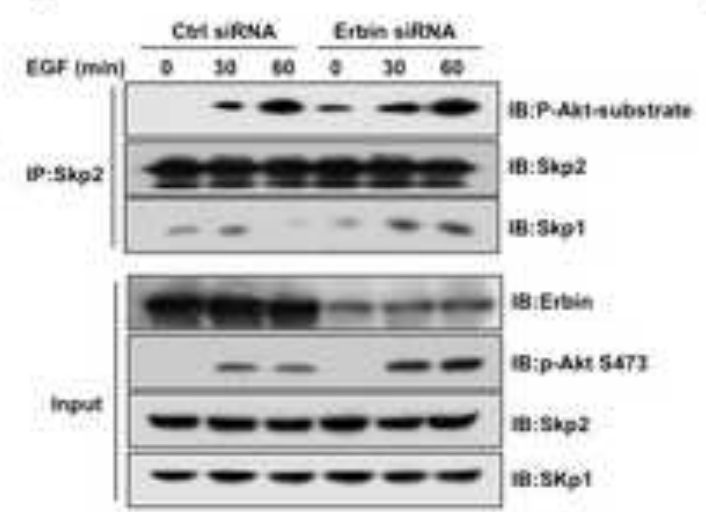

$$
\begin{aligned}
& \text { TSANAM Ctr viRKA Entin MFOA } \\
& \text { EOF omint o } 0030
\end{aligned}
$$

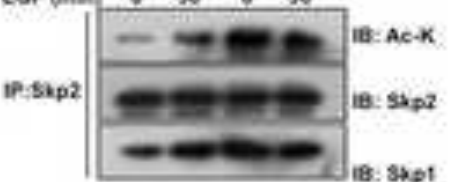

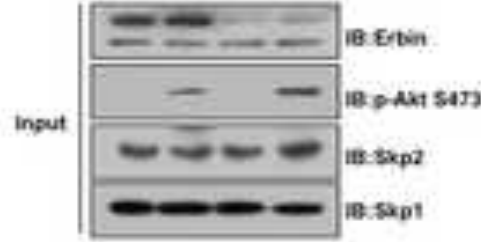


Fig. 3

A

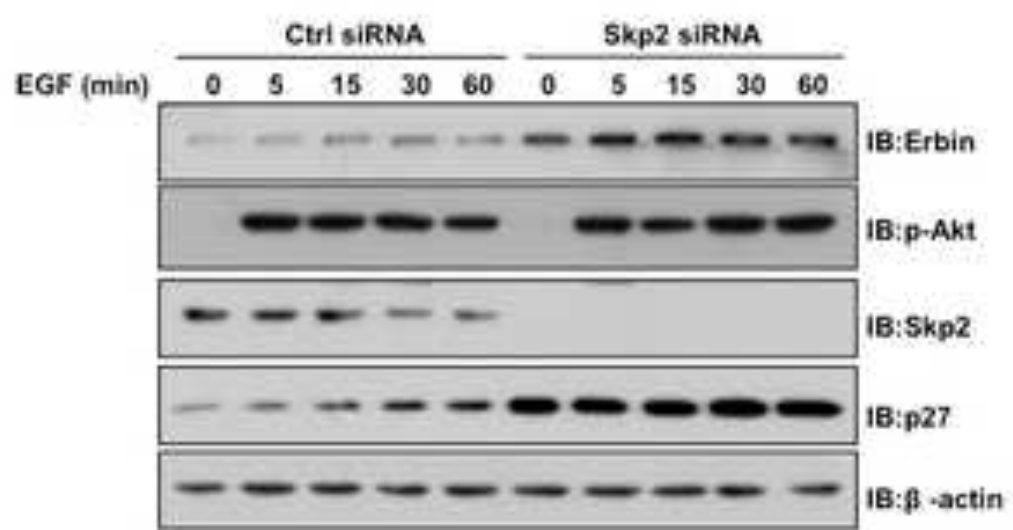

C

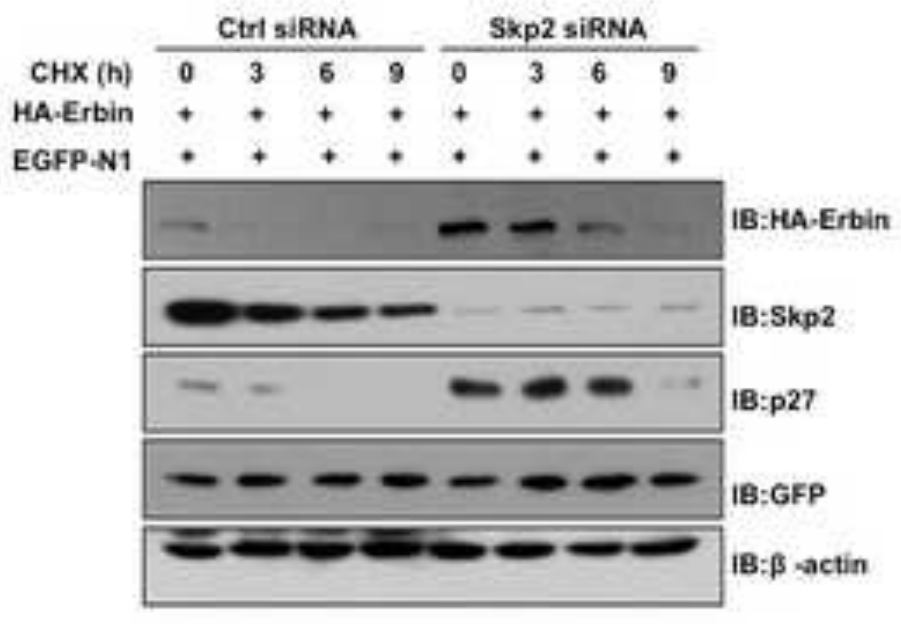

D

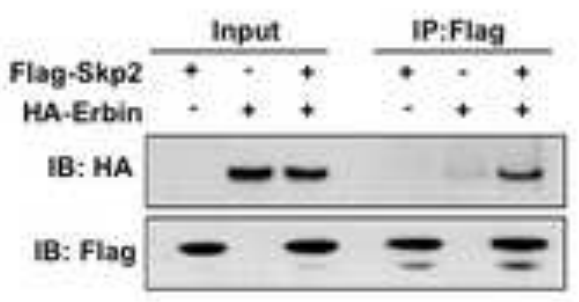

E

$\mathbf{F}$
B
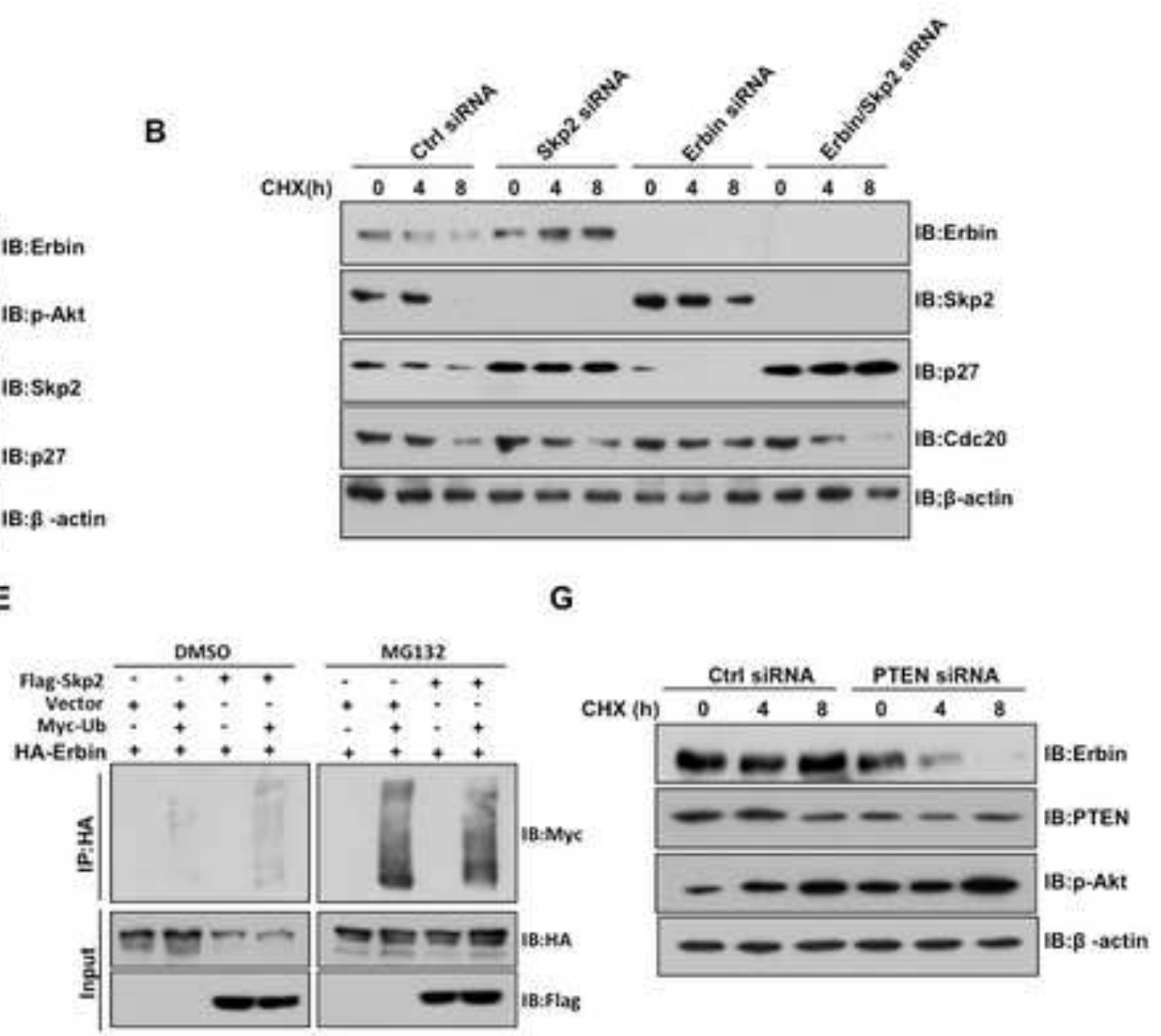

LY294002 (h) $\quad \begin{array}{llllll}0 & 3 & 6 & 9 & 12 & 24\end{array}$

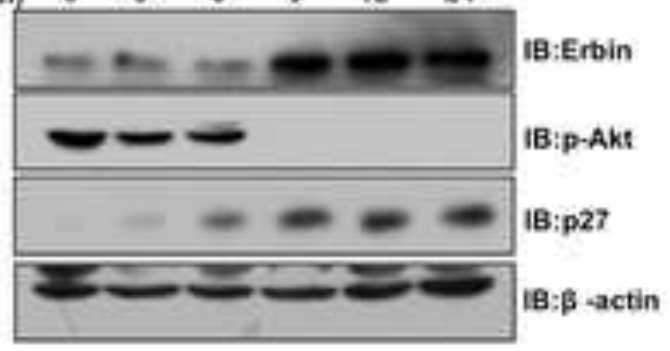


Fig. 4

A
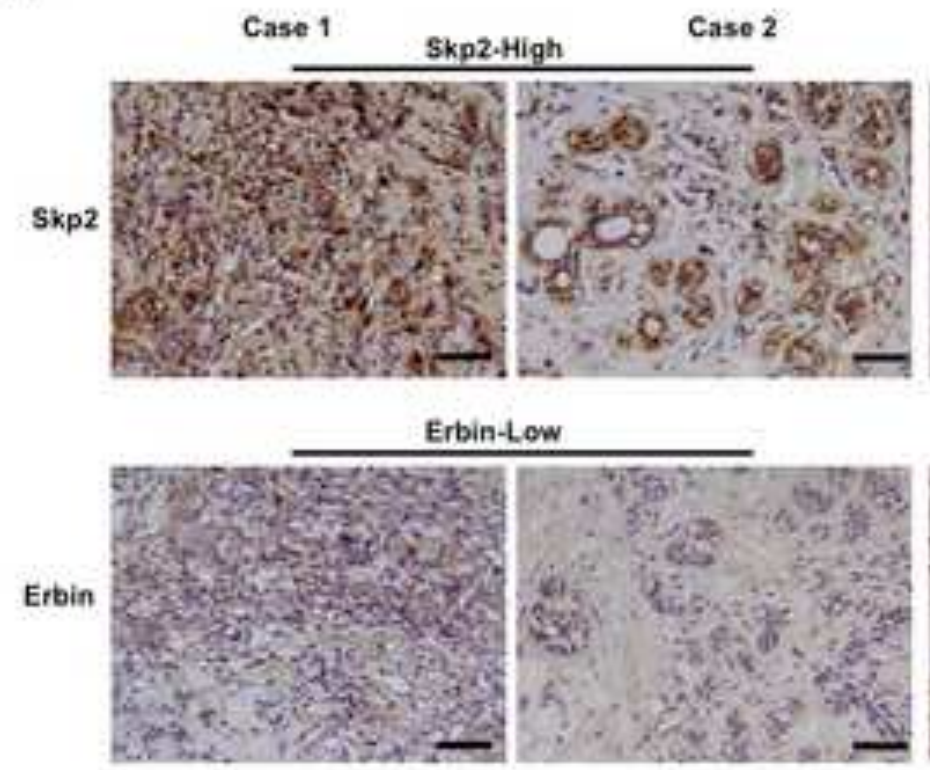

C

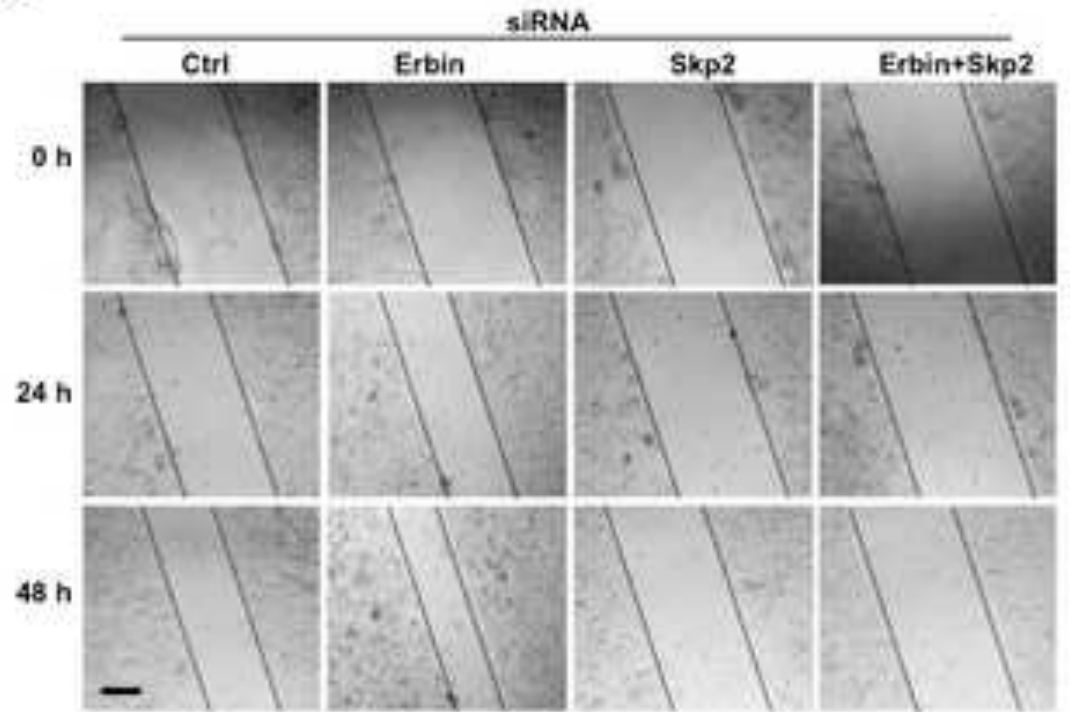

Case 1

Skp2-Low

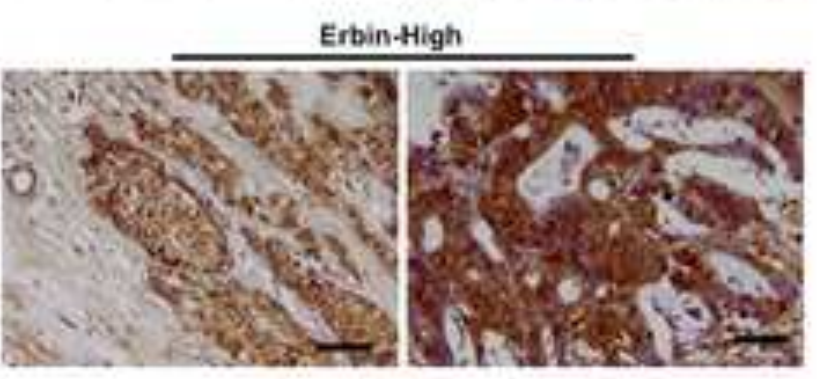

D

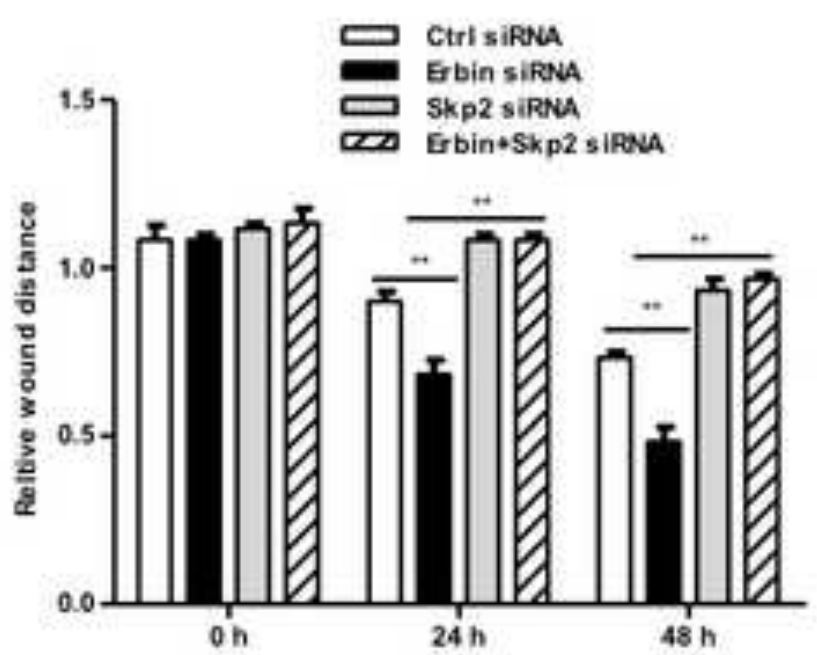

B

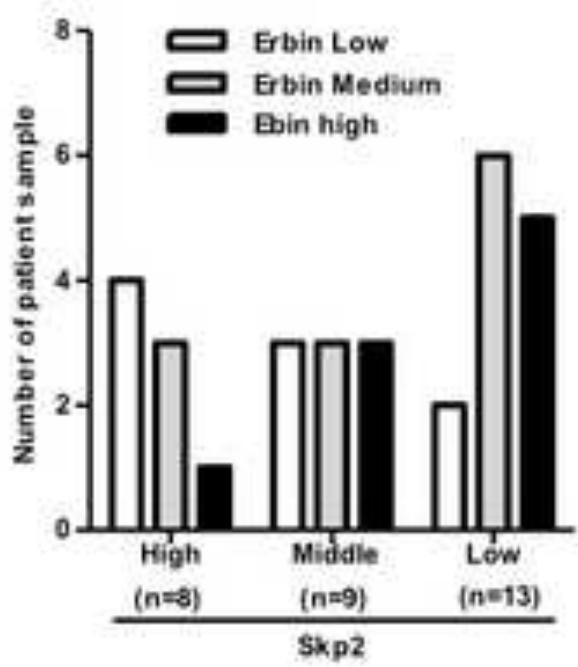

E

Oncogenic signals

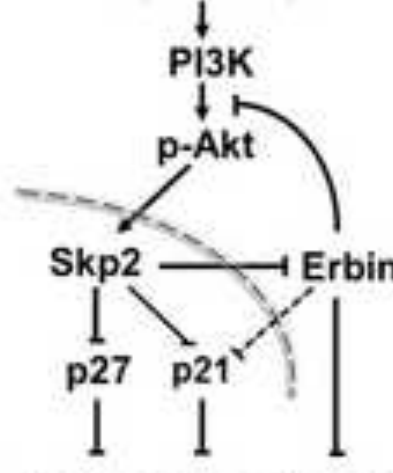

Cancer cell proliferation migration. 